\title{
Numerical Solution of Initial Value Problems Based on the Double Exponential Transformation
}

\author{
By \\ Ahniyaz Nurmuhammad*, Mayinur Muhammad* and Masatake Mori*
}

\begin{abstract}
The purpose of this paper is to present a method for approximate solution of initial value problems of ordinary differential equation by the double exponential transformation. The original problem is transformed into a Volterra integral equation and it is solved via the indefinite integration formula derived by Muhammad and Mori. A remarkable advantage of the double exponential transformation technique for solving initial value problems in this method is that it is easily implemented and gives a result with high accuracy also for problems with end point singularities and for stiff problems. The high accuracy of the method proposed in this paper is confirmed by numerical examples and an exponential convergence rate $\exp (-c N / \log N)$ is attained in almost all cases.
\end{abstract}

\section{$\S 1$. Introduction}

Suppose that a first order initial value problem of the form

$$
\left\{\begin{array}{l}
\frac{d u}{d x}=K(x, u), \quad a<x<b, \\
\left.u(x)\right|_{x=a}=u_{a}
\end{array}\right.
$$

is given. Our purpose is to solve this problem using the indefinite integration formula derived by Muhammad and Mori [2] based on the double exponential

Communicated by H. Okamoto. Received December 10, 2004. Revised April 13, 2005.

2000 Mathematics Subject Classification(s): 65L05, 65L60, 65R20.

Key Words: Double exponential transformation, DE transformation, Sinc-collocation, integral equation, initial value problem.

*Department of Mathematical Sciences, Tokyo Denki University, Hatoyama-cho, Hiki-gun, Saitama 350-0394, Japan. 
transformation. Assume that the initial value problem (1.1) has a unique solution in $(a, b)$. In order to solve it by the indefinite integration formula, we integrate the differential equation with respect to $x$ to obtain a Volterra integral equation

$$
u(x)=\int_{a}^{x} K(\xi, u(\xi)) d \xi+u_{a}, \quad a<x<b .
$$

Once the differential equation is transformed into a Volterra integral equation the double exponential indefinite integration formula proposed in [2] enables us to approximate the resulting integral equation by a system of linear or nonlinear algebraic equations, whose solution gives an approximate solution of the differential equation.

To state the results precisely, we need to define spaces of functions analytic in a strip region about the real axis, which are characterized by the decay rate of their elements in the neighborhood of the infinity. Let $\mathcal{D}_{d}$ be the strip region of width $2 d(d>0)$, i.e.

$$
\mathcal{D}_{d} \equiv\{t \in \mathbb{C}|| \operatorname{Im} t \mid<d\}
$$

Let $z=\phi(t)$ denote a conformal map of $\mathcal{D}_{d}$ onto a simply connected domain $\mathcal{D}$ with the boundary $\partial \mathcal{D}$ such that $\phi((-\infty, \infty))=(a, b)$ where $\phi(-\infty)=a$, $\phi(\infty)=b$. We denote $\phi^{-1}$ the inverse of $\phi$. Let $\mathbf{H}^{1}\left(\mathcal{D}_{d}\right)$ be the family of all functions $g$ analytic in $\mathcal{D}_{d}$ such that

$$
\begin{aligned}
& \mathcal{N}_{1}\left(g, \mathcal{D}_{d}\right)=\lim _{\varepsilon \rightarrow 0} \int_{\partial \mathcal{D}_{d(\varepsilon)}}|g(t)||d t|<\infty, \\
& \mathcal{D}_{d(\varepsilon)}=\{t \in \mathbb{C}|| \operatorname{Re} t|<1 / \varepsilon,| \operatorname{Im} t \mid<d(1-\varepsilon)\} .
\end{aligned}
$$

A function $g$ is said to decay double exponentially if there exist positive constants $\alpha$ and $C$ such that

$$
|g(t)| \leq C \exp (-\alpha \exp |t|) \quad \text { for } \quad t \in(-\infty, \infty)
$$

or a function $f$ is said to decay double exponentially with respect to the conformal map $\phi$ if there exist positive constants $\alpha$ and $C$ such that

$$
\left|f(\phi(t)) \phi^{\prime}(t)\right| \leq C \exp (-\alpha \exp |t|) \quad \text { for } \quad t \in(-\infty, \infty) .
$$

In this sense $\phi$ satisfying (1.5) is called a double exponential transformation, abbreviated as a DE transformation. Let $\mathbf{K}_{\phi}^{\alpha}\left(\mathcal{D}_{d}\right)$ denote the family of functions 
$f$ where $f(\phi(t)) \phi^{\prime}(t)$ belongs to $\mathbf{H}^{1}\left(\mathcal{D}_{d}\right)$ and decays double exponentially with respect to $\phi$ and with a constant $\alpha$ as in (1.5).

Incidentally, a function $f$ is said to decay single exponentially with respect to the conformal map $\phi_{1}$ if there exist positive constants $\alpha^{\prime}$ and $C^{\prime}$ such that

$$
\left|f\left(\phi_{1}(t)\right) \phi_{1}^{\prime}(t)\right| \leq C^{\prime} \exp \left(-\alpha^{\prime}|t|\right) \quad \text { for } \quad t \in(-\infty, \infty),
$$

and $\phi_{1}$ satisfying (1.6) is called a single exponential transformation, abbreviated as an SE transformation.

Hereafter we consider specifically the following DE transformation:

$$
\begin{aligned}
& x=\phi(t)=\frac{(b-a)}{2} \tanh \left(\frac{\pi}{2} \sinh t\right)+\frac{(b+a)}{2}, \\
& \phi^{\prime}(t)=\frac{b-a}{2} \frac{\frac{\pi}{2} \cosh t}{\cosh ^{2}\left(\frac{\pi}{2} \sinh t\right)} .
\end{aligned}
$$

Assume that $f$ is a function which belongs to $\mathbf{K}_{\phi}^{\alpha}\left(\mathcal{D}_{d}\right)$ with respect to $\phi$ defined by (1.7). Then we have the following formula for an indefinite integral based on the DE transformation [2]:

$$
\begin{aligned}
\int_{a}^{s} f(x) d x=h \sum_{j=-N}^{N} f(\phi(j h)) \phi^{\prime}(j h) & \left(\frac{1}{2}+\frac{1}{\pi} \operatorname{Si}\left(\pi \frac{\phi^{-1}(s)}{h}-j \pi\right)\right) \\
& +O\left(\frac{\log N}{N} \exp \left(-\frac{\pi d N}{\log (\pi d N / \alpha)}\right)\right),
\end{aligned}
$$

which holds uniformly for all $s \in[a, b]$ where the mesh size $h$ and the number of function evaluations $N$ should satisfy the relation

$$
h=\frac{1}{N} \log (\pi d N / \alpha) .
$$

$\operatorname{Si}(x)$ is the sine integral defined by

$$
\operatorname{Si}(x)=\int_{0}^{x} \frac{\sin \zeta}{\zeta} d \zeta .
$$

\section{$\S 2 . \quad$ Application of Indefinite Integration Formula}

We start with the first order initial value problem of the form (1.1). If we integrate (1.1) with respect to $x$ the initial value problem (1.1) can readily be transformed into an integral equation (1.2). Therefore, the formula (1.9) for 
indefinite integration can be directly applied to the first term of the right hand side of integral equation (1.2).

We assume here that the kernel $K(\xi, u(\xi))$ of the integral equation (1.2) is analytic on $a<\xi<b$ but at the end point $\xi=a$ or $\xi=b$ it may have an integrable singularity. And assume that the integral kernel $K(x, \cdot)$ belongs to $\mathbf{K}_{\phi}^{\alpha}\left(\mathcal{D}_{d}\right)$ with respect to $\phi$ defined by (1.7). Application of the indefinite integration formula (1.9) to the kernel integral in the integral equation (1.2) gives

$$
\int_{a}^{x} K\left(\xi_{j}, u(\xi)\right) d \xi \approx h \sum_{j=-N}^{N} K\left(\xi_{j}, u_{j}\right) \phi^{\prime}(j h)\left(\frac{1}{2}+\frac{1}{\pi} \operatorname{Si}\left(\pi \frac{\phi^{-1}(x)}{h}-j \pi\right)\right),
$$

where $u_{j}$ denotes an approximate value of $u\left(\xi_{j}\right)$, and

$$
\begin{aligned}
& \xi=\phi(t)=\frac{(b-a)}{2} \tanh \left(\frac{\pi}{2} \sinh t\right)+\frac{(b+a)}{2}, \\
& t=\phi^{-1}(\xi), \quad x=\phi(\tau), \quad-\infty<\tau \leq \phi^{-1}(x), \\
& \xi_{j}=\phi(j h), \quad j=-N, \ldots, N .
\end{aligned}
$$

Hereafter we call $\xi_{j}=\phi(j h), j=-N,-N+1, \ldots, N$ the Sinc points. If we replace the first term of the right hand side of (1.2) with the right hand side of (2.1) we have

$$
u(x)-h \sum_{j=-N}^{N} K\left(\xi_{j}, u_{j}\right) \phi^{\prime}(j h)\left(\frac{1}{2}+\frac{1}{\pi} \operatorname{Si}\left(\pi \frac{\phi^{-1}(x)}{h}-j \pi\right)\right) \approx u_{a} .
$$

There are $2 N+1$ unknowns $u_{j}, j=-N,-N+1, \ldots, N$ to be determined in (2.2). In order to determine these $2 N+1$ unknowns we employ the collocation method and as the collocation points we choose the Sinc points

$$
\begin{gathered}
\xi_{k}=\phi(k h)=\frac{(b-a)}{2} \tanh \left(\frac{\pi}{2} \sinh k h\right)+\frac{(b+a)}{2}, \\
k=-N,-N+1, \ldots, N .
\end{gathered}
$$

Applying the collocation to (2.2), we eventually obtain the following system of $2 N+1$ algebraic equations with $2 N+1$ unknowns $u_{j}, j=-N,-N+1, \ldots, N$ :

$$
\begin{gathered}
u_{k}-h \sum_{j=-N}^{N} K\left(\xi_{j}, u_{j}\right) \phi^{\prime}(j h)\left(\frac{1}{2}+\frac{1}{\pi} \operatorname{Si}(\pi(k-j))\right)=u_{a}, \\
k=-N,-N+1, \ldots, N .
\end{gathered}
$$


The solution $\left\{u_{j}\right\}_{j=-N}^{N}$ of the algebraic system (2.4) plays an important role in numerical solution of initial value problem (1.1) because they are approximate values of the exact solution $u(x)$ of the initial value problem (1.1) at the Sinc points $\xi_{k}=\phi(k h)$. If we need an approximate value of $u(x)$ at an arbitrary point $x$, we can use some interpolation based on the values $u_{j}$ obtained from the algebraic system (2.4). The following interpolation formula may be convenient to the present purpose:

$$
u_{N}(x)=u_{a}+h \sum_{j=-N}^{N} K\left(\xi_{j}, u_{j}\right) \phi^{\prime}(j h)\left(\frac{1}{2}+\frac{1}{\pi} \operatorname{Si}\left(\pi \frac{\phi^{-1}(x)}{h}-j \pi\right)\right) .
$$

\section{$\S 3 . \quad$ Solution of Linear Problem}

When the initial value problem is linear, i.e., the kernel of integral equation (1.2) has a form of

$$
K(\xi, u(\xi))=K(\xi) u(\xi)
$$

we can write the algebraic system (2.4) in a matrix form as

$$
\boldsymbol{u}=M \boldsymbol{u}+\boldsymbol{g},
$$

where the component of the matrix $M=\left(M_{k j}\right)$ is

$$
M_{k j}=h K\left(\xi_{j}\right) \phi^{\prime}(j h)\left(\frac{1}{2}+\frac{1}{\pi} \operatorname{Si}(\pi(k-j))\right),
$$

and the vectors $\boldsymbol{u}$ and $\boldsymbol{g}$ are

$$
\boldsymbol{u}=\left(\begin{array}{c}
u_{-N} \\
u_{-N+1} \\
\vdots \\
u_{N}
\end{array}\right) \quad \text { and } \quad \boldsymbol{g}=\left(\begin{array}{c}
u_{a} \\
u_{a} \\
\vdots \\
u_{a}
\end{array}\right)
$$

Usually we can solve the system of linear equations (3.2), i.e. $(I-M) \boldsymbol{u}=\boldsymbol{g}$, by the Gauss elimination.

Sometimes we may also be able to solve the linear system of equations (3.2) by successive approximation, that is, by means of an iterative scheme

$$
\left\{\begin{array}{l}
\boldsymbol{u}^{(0)}=\boldsymbol{g}, \\
\boldsymbol{u}^{(m+1)}=M \boldsymbol{u}^{(m)}+\boldsymbol{g}, \quad m=0,1,2, \ldots
\end{array}\right.
$$


with $\boldsymbol{u}^{(m)}$ defined as

$$
\boldsymbol{u}^{(m)}=\left(\begin{array}{c}
u_{-N}^{(m)} \\
u_{-N+1}^{(m)} \\
\vdots \\
u_{N}^{(m)}
\end{array}\right)
$$

We employed $\boldsymbol{g}=\left(u_{a}, u_{a}, \ldots, u_{a}\right)^{T}$ as an initial guess. In some cases the Seidel iteration may be used instead of the iteration scheme described in (3.5). However we do not go into details of these alternatives.

As an example we solved the following initial value problem of ordinary differential equation using the Gauss elimination and also the iteration method (3.5) mentioned above.

\section{Example 1.}

$$
\left\{\begin{array}{l}
\frac{d u}{d x}=u \sin x, \quad 0<x<1, \\
u(0)=1 .
\end{array}\right.
$$

The exact solution of this problem is $u(x)=\exp (1-\cos x)$. In order to observe the efficiency of the DE transformation we also solved this problem by the method based on the SE transformation. For the DE and SE transformations we employed

$$
x=\phi(t)=\frac{1}{2} \tanh \left(\frac{\pi}{2} \sinh t\right)+\frac{1}{2}
$$

and

$$
x=\phi_{1}(t)=\frac{1}{2} \tanh \frac{t}{2}+\frac{1}{2},
$$

respectively. For each $N$ of $N=2,4,8,16,32,64,128$ we chose the mesh size $h$ as

$$
h=\frac{1}{N} \log (\pi d N / \alpha)
$$

for the DE transformation and $h=\pi / \sqrt{N}$ for the SE transformation. Because the kernel has no singularity in $(0,1)$, we can take $d=\pi / 2$ for the DE transformation [4]. The parameter $\alpha$ is $\pi / 2$. Computation was carried out in quadruple accuracy. For each $N$, the maximum value of the absolute value of the error

$$
E_{\max }=\max _{-N \leq k \leq N}\left|u_{k}-u\left(x_{k}\right)\right|
$$

was computed at the Sinc points $x_{k}=\phi(k h)$. Also, for the SE transformation, we used the Sinc points $x_{k}=\phi_{1}(k h)$. The behavior of the error is shown in 
Fig. 1. The curve marked as DE is the error by the method based on the DE transformation and the curve marked as SE is the one based on the SE transformation. The numbers on the error curve $(8,12,16,25)$ are the times of iteration when we solved the problem by using the iteration method (3.5). The error curve corresponding to the Gauss elimination and by the iteration method (3.5) overlap almost everywhere so that they are almost indistinguishable with each other.

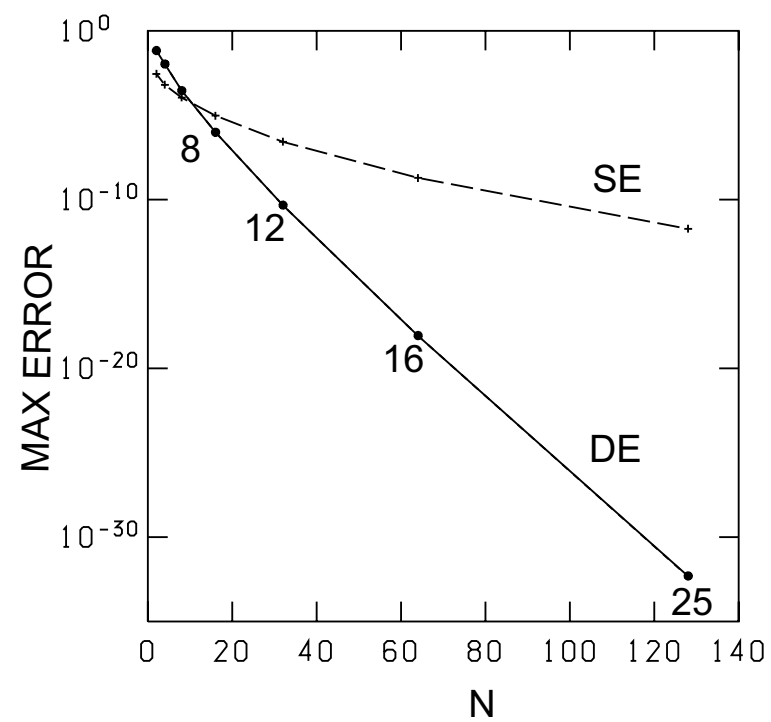

Figure 1. Max error of Example 1.

\section{§4. Solution of Nonlinear Problem}

If the initial value problem (1.1) is nonlinear, then the system of algebraic equations (2.4) is nonlinear with respect to $\left\{u_{j}\right\}_{j=-N}^{N}$. Therefore in this case, we must employ some iterative method to solve the system for $\left\{u_{j}\right\}_{j=-N}^{N}$. To solve the nonlinear algebraic system (2.4) we can successfully apply Newton's method with a good choice of the initial value in many cases. We employ here $\boldsymbol{g}=\left(u_{a}, u_{a}, \ldots, u_{a}\right)^{T}$ as an initial guess. We rewrite the algebraic system as

$$
\boldsymbol{F}(\boldsymbol{u})=\mathbf{0}
$$


using the vector $\boldsymbol{u}$ defined as (3.4) and the vector

$$
\boldsymbol{F}=\left(\begin{array}{c}
F_{-N} \\
F_{-N+1} \\
\vdots \\
F_{N}
\end{array}\right)
$$

where

$$
\begin{aligned}
& F_{k}=u_{k}-h \sum_{j=-N}^{N} K\left(\xi_{j}, u_{j}\right) \phi^{\prime}(j h)\left(\frac{1}{2}+\frac{1}{\pi} \operatorname{Si}(\pi(k-j))-u_{a},\right. \\
& \xi_{l}=\phi(l h) \quad l=-N,-N+1, \ldots, N .
\end{aligned}
$$

Then Newton's method can be written

$$
\left\{\begin{array}{l}
\boldsymbol{u}^{(0)}=\boldsymbol{g}, \\
\boldsymbol{u}^{(m+1)}=\boldsymbol{u}^{(m)}-J^{-1}\left[\boldsymbol{u}^{(m)}\right] F\left(\boldsymbol{u}^{(m)}\right), \quad m=0,1,2, \ldots,
\end{array}\right.
$$

where the component of the Jacobi matrix $J=\left(J_{k j}\right)$ is given as

$$
J_{k j}=\delta_{k j}-h \frac{\partial K\left(\xi_{j}, u_{j}\right)}{\partial u_{j}} \phi^{\prime}(j h)\left(\frac{1}{2}+\frac{1}{\pi} \operatorname{Si}(\pi(k-j))\right)
$$

and the vector $\boldsymbol{g}$ and $\boldsymbol{u}^{(m)}$ are defined as (3.4) and (3.6).

If $\boldsymbol{u}^{(m)}$ in (4.4) converges to $\boldsymbol{u}$, then we have an approximate solution of the initial value problem (1.1) at an arbitrary $x$ as

$$
u_{N}(x)=u_{a}+h \sum_{j=-N}^{N} K\left(\xi_{j}, u_{j}\right) \phi^{\prime}(j h)\left(\frac{1}{2}+\frac{1}{\pi} \operatorname{Si}\left(\pi \frac{\phi^{-1}(x)}{h}-j \pi\right)\right)
$$

similarly to $(2.5)$.

Sometimes as an alternative method to solve (2.4) we may use the following iterative scheme which is directly obtained from (2.4):

$$
\left\{\begin{array}{l}
u_{k}^{(0)}=u_{a} \\
u_{k}^{(m+1)}=u_{a}+h \sum_{j=-N}^{N} K\left(\xi_{j}, u_{j}^{(m)}\right) \phi^{\prime}(j h)\left(\frac{1}{2}+\frac{1}{\pi} \operatorname{Si}(\pi(k-j))\right) \\
k=-N,-N+1, \ldots, N-1, N, \quad m=0,1,2, \ldots
\end{array}\right.
$$

As an example, we solve the following nonlinear initial value problem of ordinary differential equation by Newton's method (4.4) and also by the iteration method (4.7). 


\section{Example 2.}

$$
\left\{\begin{array}{l}
\frac{d u}{d x}=-e^{x} u^{2}(x), \quad 0<x<1, \\
u(0)=\frac{1}{2} .
\end{array}\right.
$$

The exact solution of this problem is $u(x)=1 /\left(e^{x}+1\right)$. Since $a=0$ and $b=1$, we employ the DE transformation

$$
x=\phi(t)=\frac{1}{2} \tanh \left(\frac{\pi}{2} \sinh t\right)+\frac{1}{2} .
$$

For each number $N$ of $N=2,4,8,16,32,64,128$, we chose the mesh size $h$ as

$$
h=\frac{1}{N} \log (\pi d N / \alpha)
$$

with $d=\pi / 2, \alpha=\pi / 2$. Computation was carried out in quadruple accuracy. For each $N$, the maximum value of the absolute error

$$
E_{\max }=\max _{-N \leq k \leq N}\left|u_{k}-u\left(x_{k}\right)\right|
$$

was computed at the Sinc points $x_{k}=\phi(k h)$. To confirm the efficiency of our method, we also solved this problem by the two methods, i.e. by Newton's method (4.4) and by the simple iteration scheme (4.7) mentioned above based on the single exponential transformation $x=\phi_{1}(t)=\tanh t / 2$. The behavior of the error is shown in Fig. 2. The curve marked as DE is the error by the DE transformation and the one marked as SE is the error by the SE transformation. The numbers on the error curves $(4,4,5,5)$ and $(11,15,21,32)$ are the times of iteration when we solved the problem by using Newton's method (4.4) and by the iteration method (4.7), respectively. The error curves by Newton's method (4.4) and by the iteration method (4.7) overlap almost everywhere so that they are almost indistinguishable with each other.

\section{$\S 5 . \quad$ Stiff Problem}

Next we examined the efficiency of our method by solving the following initial value problem of a system of linear ordinary differential equations. This is a famous problem from the book by C. W. Gear [1].

\section{Example 3.}

$$
\begin{cases}\frac{d u}{d x}=998 u+1998 v, & u(0)=1 \\ \frac{d v}{d x}=-999 u-1999 v, & v(0)=0 .\end{cases}
$$




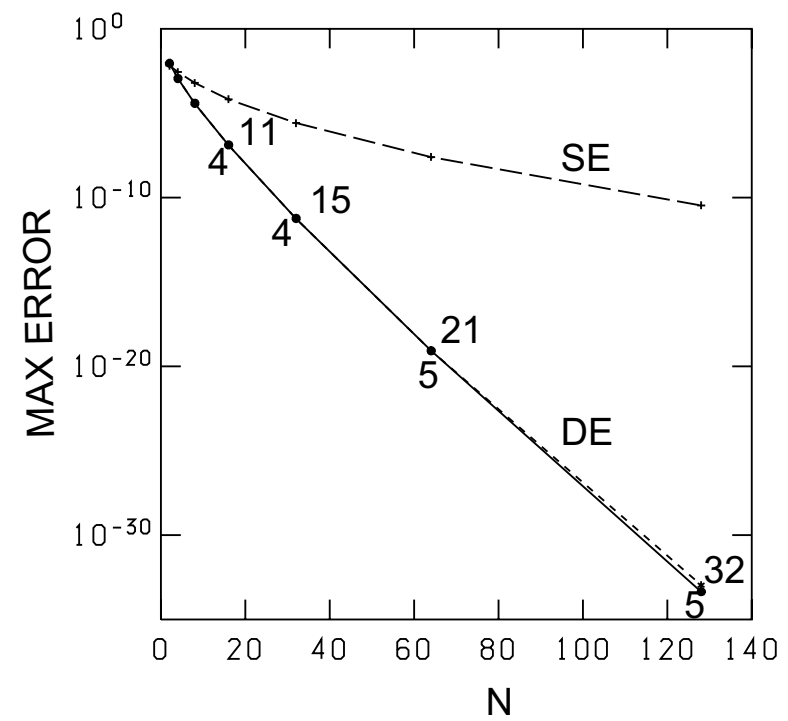

Figure 2. Max error of Example 2.

The exact solution is

$$
\left\{\begin{array}{l}
u(x)=2 e^{-x}-e^{-1000 x} \\
v(x)=-e^{-x}+e^{-1000 x}
\end{array}\right.
$$

This system of equations is regarded as a typical example of stiff problems. To solve this equation by means of the procedure mentioned in the previous section, we employ the DE transformation

$$
x=\exp \left(t-e^{-t}\right)
$$

presented in [4] because the present problem is defined on $0 \leq x<\infty$. For each $N$ of $N=2,4,8, \ldots, 256$ we chose the mesh size $h$ such that it satisfies the relation

$$
h=\frac{1}{N} \log (\pi N / 4),
$$

and the following maximum error $E_{\max }$ at the Sinc points was computed:

$$
E_{\max }=\max _{-N \leq j \leq N}\left\{\left|u\left(x_{j}\right)-u_{j}\right|,\left|v\left(x_{j}\right)-v_{j}\right|\right\}, \quad x_{j}=\phi(j h) .
$$


We also solved this problem using the following transformation and the mesh size [3]:

$$
x=\log \left(e^{t}+\sqrt{e^{2 t}+1}\right), \quad h=\frac{\pi}{\sqrt{2 N}} .
$$

This gives an SE transformation. The error curves marked as DE and as SE in Fig. 3 are the results. We should note that, although we did not pay any special care for the stiffness, the DE transformation is very efficient for this stiff problem as seen from Fig. 3 .

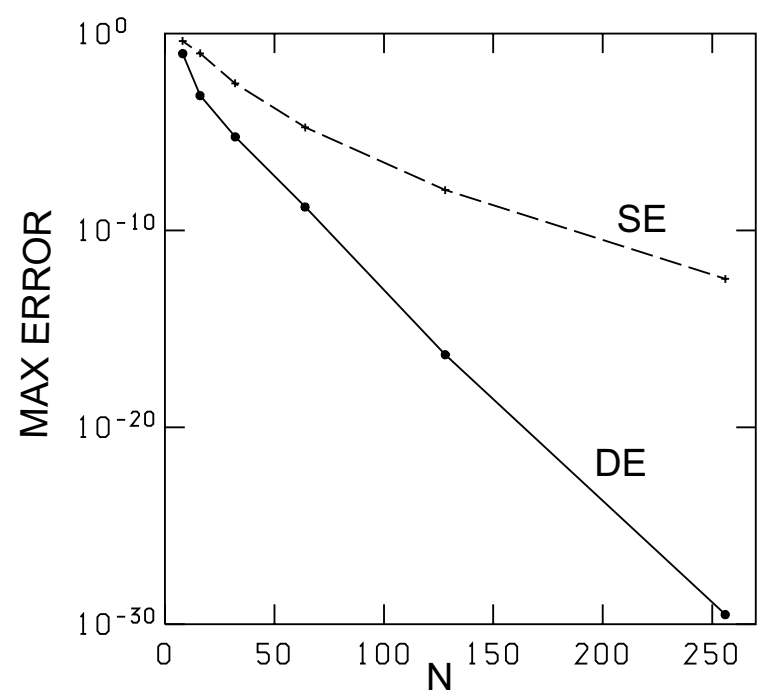

Figure 3. Max error of Example 3.

In every examples shown above error behavior which can be regarded as $O(\exp (-c N / \log N))$ is observed when we used the DE transformation. This is a direct result from (1.9) in case of linear problems [2]. Although we proposed simple iteration schemes (3.5) and (4.7) in order to solve the algebraic system, both schemes are not always guaranteed to converge to the exact solution. Therefore, we should be careful when we solve the problem using these iteration schemes. We recommend Newton's method described in (4.4) for nonlinear problems. To investigate the convergence conditions of Newton's method as well as of the iteration schemes (3.5) and (4.7) will be left to the future work.

Finally the authors are grateful to the referee for valuable comments. 


\section{References}

[1] Gear, C. W., Numerical Initial Value Problems in Ordinary Differential Equations, Prentice-Hall, Inc. Englewood Cliffs, New Jersey, 1971.

[2] Muhammad, M. and Mori, M., Double exponential formulas for numerical indefinite integration, J. Comput. Appl. Math., 161 (2003), 431-448.

[3] Stenger, F., Numerical Methods Based on Sinc and Analytic Functions, Springer-Verlag, Berlin, New York, 1993.

[4] Takahasi, H. and Mori, M., Double exponential formulas for numerical integration, Publ. RIMS, Kyoto Univ., 9 (1974), 721-741. 